\title{
Propuesta teórico-metodológica para analizar las representaciones sobre migrantes y nacionales*
}

\section{A Theoretical-Methodological Proposal for the Analysis of Representations of Migrants and Nationals}

\author{
Gisele Kleidermacher $^{* *}$ \\ Anahí González ${ }^{* * *}$ \\ Universidad de Buenos Aires, Argentina.
}

\section{Resumen}

El presente trabajo tiene como objetivo presentar el análisis realizado respecto a las representaciones sociales de encuestados/as nacidos/as y residentes en la Ciudad Autónoma de Buenos Aires acerca de la identidad nacional argentina y sobre la población de origen paraguayo y boliviano que reside en la Ciudad Autónoma de Buenos Aires. Para dicho propósito, se seleccionaron, en el marco de un cuestionario estructurado aplicado a 400

\footnotetext{
* La información analizada en el presente artículo ha sido producida en el marco del proyecto UBACYT (Subsidio de la Universidad de Buenos Aires para Ciencia y Técnica), titulado: "Los puentes entre el poder judicial, la institución educativa y la sociedad civil ante la diversidad etno-nacional en el AMBA", con sede en el IIGG. Proyecto de Investigación Científica, de Innovación Tecnológica e Interdisciplinarios. Programación Científica 2014-2017, financiado por la Universidad de Buenos Aires, Argentina.

** Licenciada en Sociología y doctora en Ciencias Sociales, Universidad de Buenos Aires (UBA). Investigadora del Consejo Nacional de Investigaciones Científicas y Técnicas (CONICET)-Instituto de Investigaciones Gino Germani, UBA. Dirección postal: Uriburu 9506 Piso, Of. 24. CABA, Argentina. Correo electrónico: kleidermacher@ gmail.com, ORCID: https://orcid.org/0000-0001-8739-8653

*** Licenciada en Sociología y doctora en Ciencias Sociales, UBA. Investigadora del Instituto de Investigaciones Gino Germani, Facultad de Ciencias Sociales, UBA. Dirección postal: Uriburu 9506 Piso, Of. 24. CABA, Argentina. Correo electrónico: anahipgonzalez@gmail.com, ORCID: https://orcid.org/00000001-5074-5260
}

Cómo citar este artículo: Kleidermacher, G. y González, A. (2020). Propuesta teórico-metodológica para analizar las representaciones sobre migrantes y nacionales. Si Somos Americanos. Revista de Estudios Transfronterizos, 20(2), 38-66. doi: 10.4067/S0719-09482020000200038 
casos, tres preguntas de carácter abierto. Como parte del presente escrito, se presenta una reflexión respecto al cierre de las mismas, que consta de un proceso de construcción de categorías conceptuales, conformadas a partir del proceso de codificación abierto y axial siguiendo la teoría fundamentada. Entre los principales hallazgos, encontramos que los migrantes, tanto paraguayos como bolivianos, son representados desde una lógica economicista e instrumental, mientras que la identidad nacional, construida en oposición a la "otredad" migrante, se compone de elementos tomados del pasado y del futuro, que actúan como aglutinadores de una identidad simbólica.

Palabras clave: representaciones sociales, migrantes, metodología.

\begin{abstract}
The goal of this paper is to analyze the social representations of people who were born and live in the Autonomous City of Buenos Aires. This focus is the national identity of Argentina and the social representations of the Paraguayan and Bolivian population in said city. Three open-ended questions from a structured questionnaire applied to 400 cases were used to inform the research. The author focuses on the closure of those questions, which consists of a construction process of conceptual categories, formed from the open and axial coding process in accordance with the Grounded Theory. The main findings suggest that Paraguayan and Bolivian migrants are represented in terms of an economic and instrumental logic while the national identity, which was built in opposition to the migrant "otherness," consists of elements of the past and the future, which act as binders of a symbolic identity.
\end{abstract}

Keywords: social representations, migrants, methodology.

\title{
Introducción
}

El presente trabajo se inserta en las líneas de investigación de un equipo de cientistas sociales que desde la década de 1990 se encuentra indagando sobre el fenómeno de la desigualdad en el marco de las relaciones interculturales. En este marco, y con un fondo de financiación de la Universidad de Buenos Aires, se ha llevado a cabo la presente investigación con enfoques cualitativo y cuantitativo que aporta datos que aquí analizamos. Bajo el título de "Los puentes entre el poder judicial, la institución educativa y la sociedad civil ante la diversidad etno-nacional en el AMBA", esta se propone indagar en los vínculos que se establecen entre el desempeño institucional de la escuela y el poder judicial

\footnotetext{
${ }^{1}$ Área Metropolitana de Buenos Aires.
} 
con la sociedad civil, ante la cuestión intercultural y las estrategias relacionales entre los diferentes actores, focalizándose en las representaciones sociales acerca de la población migrante llegada a partir de la segunda mitad del siglo XX al AMBA.

Nuestra propuesta se basa en la construcción de categorías conceptuales, conformadas a partir del análisis y codificación de las respuestas obtenidas tras la aplicación de un cuestionario semiestructurado que combina preguntas abiertas y cerradas. El instrumento de registro fue aplicado a una muestra no probabilística, compuesta por 401 casos, conformada por hombres y mujeres de entre 25 y 65 años, todos ellos residentes, al momento de la encuesta, en la Ciudad Autónoma de Buenos Aires.

Del total del cuestionario, hemos seleccionado tres preguntas de respuesta abierta, pertenecientes al primer módulo que refería a la construcción de la identidad nacional y la mirada sobre las migraciones al país. Las dos primeras preguntas consistían en elegir una palabra para describir "cómo son" los migrantes bolivianos y paraguayos que viven en la Argentina. La tercera pregunta interrogaba acerca de cuál es, según la opinión del encuestado o encuestada, la principal característica de "ser argentino".

Dado que se trata de un instrumento de registro estructurado, en el marco de una aproximación cuantitativa, el objetivo del artículo ha sido analizar la distribución de frecuencias que se concentran en cada respuesta para aprehender cuáles habían sido las tendencias discursivas ${ }^{2}$ de los/as entrevistados/as. Sin embargo, dada la diversidad y multiplicidad de respuestas, el desafío que se planteó fue el cierre y agrupamiento de estas.

Para sortear este obstáculo, planteamos la siguiente estrategia: agrupar las respuestas sobre la base de criterios de similitud, ${ }^{3}$ entre ellos, aquel que de acuerdo a la teoría fundamentada (Glaser y Strauss, 1967; Strauss y Corbin, 2002) correspondería a la etapa de "codificación abierta". Posteriormente, buscamos criterios de similitud y diferenciación al interior de esta primera agrupación, correspondiente a la etapa de "codificación axial". Finalmente, establecimos nuevos nombres para las categorías finales, considerando tanto las respuestas obtenidas como el marco teórico de las investigaciones que hemos venido desarrollando sobre la temática.

Una vez determinadas las categorías finales y renombradas, se prosiguió al análisis de la distribución de frecuencias de respuestas acumuladas en dichas categorías, identificando regularidades en los discursos. Entendemos, de este modo, que el proceso desarrollado tiene una relación inseparable entre la metodología y la teoría que cuestiona, en ese sentido, las diversas propuestas de desarrollo de índices como construcciones teóricas disociadas de la "realidad", o bien, como producto de "observaciones empíricas" desprovistas de teoría.

\footnotetext{
${ }^{2}$ Nos referimos a las regularidades en los discursos de los/as encuestados/as en relación con la caracterización de los distintos grupos nacionales.

${ }^{3}$ La etapa de codificación abierta del método de teoría fundamentada procura "abrir" el texto, buscando descubrir, nombrar y desarrollar los conceptos, exponiendo los pensamientos e ideas, y los significados. Aquí es clave, para su validación, la comparación continua de los datos.
} 
La especificidad de nuestra propuesta radica en que tomamos los aportes de la tipología construida, tal como los describe Mckinney (1968), combinándolos con la estrategia de análisis de la teoría fundamentada.

Entre los principales hallazgos podemos distinguir aquellos que refieren a lo que consideramos las representaciones sociales más significativas de los nativos acerca de los colectivos analizados (paraguayos, bolivianos y los mismos argentinos) y aquellos resultados que remiten a la potencialidad que la perspectiva teórico-metodológica, asumida para la construcción del dato, ha significado. Dentro del primer tipo de hallazgo podemos afirmar que los paraguayos son sujetos representados por los/as encuestados/as como desviados de la norma: violentos y problemáticos. En cambio, la mirada acerca de los bolivianos ha remitido a su etnitización y vinculación con la sumisión y aceptación pasiva de la explotación. Asimismo, unos y otros colectivos son asimilados a la categoría de "trabajadores", es decir, su identidad se encuentra estrechamente relacionada con la pertenencia al sector del trabajo y fundamentalmente a ser colocados, a nivel representacional, en el rol de competencia (desleal) de la mano de obra nacional. En relación con las representaciones sociales sobre los argentinos, los/as encuestados/as construyen una idea de comunidad nacional basada en pautas culturales y elementos idiosincráticos que refuerza la pertenencia al colectivo. Por otra parte, se mencionan como atributos relevantes el ser "abiertos", pero también "arrogantes" e "individualistas".

En cuanto al segundo tipo de hallazgos, pensamos que ha sido un acierto abordar el tema en cuestión a partir del análisis de las representaciones sociales y colocar en tensión algunos de los postulados de posiciones que sostienen una marcada preferencia por el estudio de las representaciones sociales desde un enfoque cualitativo. Esa postura cualitativista se evidencia en que la mayor cantidad de investigaciones, al menos en Latinoamérica, asumen dicha perspectiva en sus estrategias metodológicas (Aliaga, Maric y Uribe, 2018). En el Cuadro $\mathrm{N}^{\circ} 1$ se resumen estas dos posturas, sus elementos fundamentales y sus autores más representativos, basándonos en el trabajo de Banchs (2000). En su artículo, esta autora propone la clasificación entre abordajes estructurales y procesuales, no sin aclarar -aspecto con el que también coincidimos- que estos dos tipos de enfoques son abstracciones que seguramente no se darán de forma pura, pero que permiten afirmar que los estudios sobre representaciones sociales pueden agruparse a lo largo de un continuиm en el cual en uno de sus polos estaría el enfoque procesual y, en el otro, el enfoque estructural (Banchs, 2000) pudiendo, en la práctica investigativa, existir estrategias teórico-metodológicas más o menos cercanas a cada uno de estos extremos.

El polo procesual es definido por esta autora como aquel "que va más allá del Interaccionismo Simbólico, hacia una postura socioconstruccionista, ciertamente originada a partir de los postulados interaccionistas y claramente influenciada por la literatura foucaultiana, sobre todo en términos de análisis del discurso"; en cambio, "el polo estructural es aquel que se focaliza sobre la estructura de las representaciones sociales, 
haciendo uso del método experimental o bien sofisticados análisis multivariados que permiten identificar esta estructura" (Banchs, 2000, p. 3.5).

\section{Cuadro $N^{\circ}$ 1: Comparación de enfoques procesual y estructural}

\begin{tabular}{|c|c|c|}
\hline & Enfoque procesual & Enfoque estructural \\
\hline Principales postulados & $\begin{array}{l}\text { Abordaje hermenéutico. } \\
\text { Los sujetos son productores de } \\
\text { sentidos. } \\
\text { El lenguaje construye el mundo } \\
\text { en el que vivimos. } \\
\text { Análisis de las producciones } \\
\text { simbólicas de los significados } \\
\text { del lenguaje. } \\
\text { Los significados están ligados al } \\
\text { contexto histórico y cultural. }\end{array}$ & $\begin{array}{l}\text { Identificar el núcleo de la } \\
\text { representación social para } \\
\text { desarrollar explicaciones } \\
\text { sobre la estructura. } \\
\text { Conocer la organización de } \\
\text { los elementos de la } \\
\text { representación social: núcleo } \\
\text { central y elementos } \\
\text { periféricos. } \\
\text { Identificar las jerarquías } \\
\text { entre los diversos elementos } \\
\text { de la representación social. }\end{array}$ \\
\hline Método utilizado & $\begin{array}{l}\text { Cualitativo y triangulación de } \\
\text { múltiples técnicas }\end{array}$ & 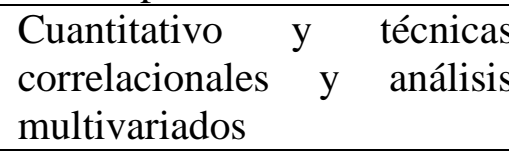 \\
\hline Tipo de estudio & Procesual & Estructural/Estático \\
\hline Objetivo & $\begin{array}{l}\text { Comprender los hechos } \\
\text { singulares que generan una } \\
\text { representación social. }\end{array}$ & $\begin{array}{l}\text { Conocer la organización de } \\
\text { la representación social } \\
\text { particular a partir de sus } \\
\text { elementos constituyentes. }\end{array}$ \\
\hline Autores principales & Moscovici, Jodelet & Abric, Rateau, Lo Monaco \\
\hline
\end{tabular}

Fuente: elaboración propia sobre la base de Banchs (2000).

La elección de una u otra postura dependerá de los objetivos de cada investigación. Las decisiones metodológicas que hemos tomado en varias de nuestras investigaciones nos han acercado más a la postura procesual; no obstante, en esta oportunidad el cuestionario incluye algunas de las técnicas y propósitos atribuidos al enfoque estructural. En este sentido, somos conscientes de que la introducción de una técnica cuantitativa suele asociarse con este tipo de posturas, pero partimos de una mirada epistemológica no dicotómica entre métodos cualitativos y cuantitativos.

Así, la mixtura de enfoques ha permitido construir y ordenar los datos (cualitativos y cuantitativos), de modo de hacerlos comparables, al tiempo que el peso relativo de una y 
otra representación ha podido ser ponderado, posibilitándose así una mejor comprensión y aprehensión del fenómeno bajo estudio.

El artículo se estructura del siguiente modo: en primer lugar, se realizan algunas precisiones teóricas referentes a la categoría de representación social como herramienta analítica. A continuación, presentamos un apartado metodológico, en el que abordamos los principales aportes recogidos de Glaser y Strauss (1967) en relación con la teoría fundamentada, y la construcción de categorías de análisis de Mckinney (1968) en relación con la construcción de tipologías. Posteriormente, se presentan los resultados, es decir, el análisis de las representaciones sociales de la población nativa respecto de los inmigrantes paraguayos y bolivianos para, enseguida, analizar la autopercepción de los propios argentinos. En cada uno de los apartados donde se analizan los resultados se encuentran dos cuadros, el primero presenta una codificación más abierta, mientras que en un segundo cuadro se presenta un cierre mediante la conformación, en algunos casos, de nuevas categorías. Consideramos que de este modo es posible visibilizar el trabajo de codificación en sus diversos niveles. Finalmente, se presentan las conclusiones a modo de cierre.

\section{Algunas precisiones teóricas}

Como hemos anticipado, el material que se analizará en este escrito se desprende de una investigación de mayor amplitud. También podemos afirmar que es la continuación de una línea de trabajo desarrollada durante varios años, en la que hemos abordado la temática de la interculturalidad a partir del estudio de las representaciones sociales. Partimos de una idea de las representaciones entendidas como elementos centrales en la construcción de la realidad social (Berger y Luckmann, 2006).

Asimismo, la relevancia del concepto de representaciones sociales radica en que relaciona los procesos simbólicos con las conductas, en tanto y en cuanto, en las representaciones tiene lugar un proceso de elaboración cognitiva y simbólica que orienta los comportamientos (Berger y Luckmann, 2006; Jodelet, 1991; Moscovici, 1986). En este sentido, Jodelet (1991) vincula la existencia de las representaciones sociales con la necesidad que tienen todos los sujetos de saber a qué atenerse ante el mundo que los rodea, es decir, a la previsibilidad que se requiere para conducirnos en el mundo social, de manera que las representaciones sociales “(...) nos orientan en la manera de designar y definir conjuntamente los diferentes aspectos de nuestra realidad diaria, en la manera de interpretarlos, influir sobre ellos" (Jodelet, 1991, p. 203).

En este proceso de hacer a la "realidad" previsible y reducir la incertidumbre es que se generan modos de calificación y clasificación de los “otros". Estos otros pueden ser más o menos cercanos. También se establecen diferencias que, en sí mismas, no implican

desigualdades. Sin embargo, estas pueden surgir cuando las clasificaciones son 
jerarquizantes. A partir de allí, las representaciones sociales no resultan ya inocuas recetas para la acción -reactualizándose y anclándose en las imágenes, pensamientos e ideas propias del sentido común-, sino que estructuran relaciones de dominación. En el presente artículo nos interesa considerar las representaciones sociales como una herramienta analítica que nos posibilita aprehender este proceso de constitución de un "nosotros" nativo y de un "otro" extranjero a partir de las formas que proponen los/as entrevistados/as para categorizar lo "propio" y lo "ajeno" respecto a la comunidad nacional.

Consecuentemente, nuestro análisis se complejiza porque empleamos, como herramienta conceptual, las representaciones para abordar una temática sensible que refiere a aquellas ideas e imágenes que construimos los nativos en relación con la otredad migrante. Como dijimos, este cúmulo de sentidos no refiere tan solo a representaciones ordenadoras de la realidad social, sino que implica relaciones de dominación y de poder (Carballude, Malegaríe, Pérez y Santamaría, 2004), supone conceptualizar, nominar, calificar y clasificar no solo sucesos y objetos, sino también a personas e incluso a colectivos de personas, como ocurre en el caso de los estereotipos. Es aquí que retomamos otras categorías de autores que se han preguntado acerca de procesos excluyentes como la discriminación y el racismo (Caggiano, 2005; Grimson, 2013; Van Dijk, 1997), y la constitución de identidades nacionales (Anderson, 1993; Balibar, 2005; Hall, 1996; Vernik, 2004) y su articulación con una idea ficcional edificada a partir de una serie de atributos y elementos culturales considerados esencialmente propios de un colectivo aglutinado alrededor de un Estado que es, en última instancia, el que define quiénes formarán parte de la comunidad de nativos. Escapa a las posibilidades y a la extensión del artículo describir las teorías de cada uno de estos autores; no obstante, resulta pertinente explicitar su influencia aquí, ya que serán retomados a lo largo del análisis de los resultados de la investigación. ${ }^{4}$

Teniendo presente estos conceptos -discriminación, relaciones de dominación y construcción de una identidad nacional- que son parte nodal del marco teórico de la investigación, comenzaremos en las páginas que siguen con el análisis de las representaciones sociales que los encuestados/as construyen acerca de paraguayos, bolivianos y de sí mismos como argentinos.

\footnotetext{
${ }^{4}$ Hemos desarrollado esta temática más extensamente en el artículo de nuestra autoría (González y Kleidermacher, 2018).
} 


\section{Metodología $^{5}$}

Con relación a la estrategia metodológica utilizada, se combinaron elementos de la teoría fundamentada y la construcción de índices. En un intento de superación de la dicotomía entre las estrategias cualitativas y cuantitativas, respectivamente, nos propusimos construir conceptos a partir de las preguntas abiertas planteadas en el cuestionario, que posteriormente han sido utilizadas para contabilizar las frecuencias porcentuales concentradas en cada una de ellas.

Retomando a Mckinney (1968), entendemos que el tipo "puede ser usado como concepto generalizador por medio del cual uno puede extractar sus versiones empíricas de diferentes contextos culturales" (p. 31). Asimismo, el autor plantea que conceptuar significa generalizar hasta cierto grado y, a la vez, generalizar significa reducir el número de objetos concibiendo algunos de ellos como idénticos. En este sentido hemos procedido a realizar un doble ejercicio de apertura y cierre de conceptos, tal como lo plantean Glaser y Strauss (1967). Estos autores sostienen lo que denominan "método de comparación constante", el que refiere a un proceso que consta de diferentes etapas, la primera de las cuales consiste en la "codificación abierta", en que los datos se descomponen en partes discretas, se examinan minuciosamente y se comparan en busca de similitudes y diferencias. Los acontecimientos, sucesos, objetos y acciones o interacciones que se consideran conceptualmente similares en su naturaleza o relacionados en el significado, se agrupan bajo conceptos más abstractos, denominados "categorías" (Glaser y Strauss, 1967, pp. 111-112).

De este modo, tal como expresan Glaser y Strauss (1967), en esta primera etapa "el analista comienza por codificar cada incidente dentro de tantas categorías de análisis como sea posible, sea que surjan categorías o sea que los datos surjan y encajen en categorías existentes" (p. 35). ${ }^{6}$ Una segunda etapa es la que denominan "codificación axial", centrada en la codificación y búsqueda de relaciones a nivel de las categorías. Tal como plantean Strauss y Corbin (2002), "en la codificación axial, las categorías se relacionan con sus subcategorías para formar unas explicaciones más precisas y completas sobre los fenómenos" (2002, p. 135). Es decir, se busca enlazar los datos según sus dimensiones y propiedades "para formar categorías densas, bien desarrolladas y relacionadas entre sí" (Strauss y Corbin, 2002, p. 135).

\footnotetext{
${ }^{5}$ Los/as encuestados/as que participaron del relevamiento fueron previamente informados acerca de que los objetivos de la investigación respondían a fines estrictamente académicos, garantizándoseles el anonimato y la confidencialidad de sus datos personales, a partir de lo cual manifestaron su conformidad a cooperar en el proceso de la encuesta.

${ }^{6}$ Cabe destacar, en términos de Strauss y Corbin (2002), que "las categorías son conceptos derivados de los datos que representan fenómenos. El analista puede poner nombre a causa de la imagen o significado que evocan cuando los examina comparativamente y en el contexto, o el nombre se puede tomar de las palabras de los entrevistados mismos, estos son los códigos in vivo o emic" (p. 110). A su vez, expresan que "las propiedades son las características generales o específicas o los atributos de una categoría, cuya delineación la define y le da significado. Las dimensiones son la escala en la cual varían las propiedades generales de una categoría y que le da especificaciones a la categoría y variaciones a la teoría" (Strauss y Corbin, 2002, p. 110).
} 
A su vez, es importante señalar, tal como sostienen Glaser y Strauss (1967), que "cuando las categorías y sus propiedades emergen, el analista descubre dos clases: aquellas que él ha construido por sí mismo y aquellas que han sido abstraídas del lenguaje de la situación de investigación" (p. 35). De esta forma queda de manifiesto la construcción del "dato" a partir de la imbricación teórico-metodológica. También Mckinney (1968) refiere a este proceso al que denomina "tipos construidos", a los que se les da precisión por medio de la selección, limitación, combinación y acentuación. Los tipos construidos "ordenan una serie de atributos en una configuración que no se experimenta necesariamente en forma directa y da énfasis a uno o varios de los atributos con fines teóricos. Su valor se debe medir en términos de su capacidad explicativa" (Mckinney, 1968, p. 99).

Una de las funciones científicas del tipo construido es el ordenamiento de los datos concretos de manera que puedan ser descritos en términos que los hagan comparables (Glaser y Strauss, 1967). Es una construcción formada por elementos abstractos e integrada en un modelo conceptual unificado en donde puede haber intensificación de uno o más aspectos de la experiencia concreta.

Cabe destacar que las tipologías están subordinadas a los fines de la investigación, es decir, al establecimiento de uniformidades de valor explicativo. Las tipologías son instrumentales en el proceso de investigación; son ficciones, ya que han sido construidas. De esta forma, y a partir de la construcción de dichos conceptos, hemos procedido a su medición para posterior comparación y análisis del peso relativo que cada uno de ellos tiene en las representaciones de los actores encuestados.

\section{Resultados}

Los resultados del presente trabajo se presentan a continuación separados por secciones en torno a cada uno de los grupos migratorios con los que trabajamos, dado que cada uno de ellos contiene sus propias particularidades, mientras que en el último apartado se presentan las conclusiones tomadas como conjunto.

\section{Representaciones sociales sobre paraguayos}

El acto de nominar a la otredad supone un ejercicio de poder que incide en el modo en que los miembros de la sociedad hospitante, en su calidad de "establecidos" (Elias, 2003), trazan fronteras y puentes a nivel representacional con los extranjeros. Antes de analizar las representaciones sociales y, consecuentemente, dichos límites y vínculos que a nivel simbólico los nativos construyen acerca de los migrantes paraguayos, resulta imperioso comentar, aunque más no sea de modo somero y solo con fines de contextualización, 
algunas de las características que la migración paraguaya ha asumido en el territorio argentino.

El primer dato a señalar es que se trata de la comunidad migrante de mayor presencia numérica. País limítrofe de Argentina, los contactos entre ambas naciones, sobre todo en las zonas de frontera, han sido constantes a lo largo de la historia. Siguiendo datos censales, Halpern (2009) señala que ya en 1869 constituían el 0,2\% de la población total del país. Asimismo, representaban el 1,6\% sobre la población extranjera y el 7,9\% sobre los migrantes de países limítrofes. Recordemos que hasta principios del siglo XX, la presencia numérica de mayor incidencia entre los extranjeros estaría compuesta por los provenientes de Europa, fundamentalmente italianos y españoles (Devoto, 2003). Con el correr de las décadas, se produjo una mejoría en la cuantificación de las migraciones regionales que, junto a un real aumento en relación con las migraciones de ultramar, irán constituyendo a los paraguayos en la colectividad con mayor presencia en términos porcentuales. Así, a partir de 1947, es el grupo más numeroso entre las migraciones latinoamericanas.

Este incremento se sostiene, de acuerdo al último Censo Nacional realizado en 2010. Su volumen asciende a 550.713 personas de origen paraguayo residentes en la República Argentina (INDEC, 2010) ${ }^{7}$. Si bien estos números son ínfimos en relación con la cantidad de habitantes de Argentina (40.117.096, según el último Censo Nacional de Población), toman notoriedad en la Provincia de Buenos Aires y la Ciudad Autónoma (centros industriales), lugares a los que por lo general suelen dirigirse, fundamentalmente, en búsqueda de puestos de trabajo (Halpern, 2009).

Habiendo efectuado esta resumida contextualización de las migraciones paraguayas en la Argentina, analizaremos ahora la información de la encuesta realizada a los nativos acerca de las construcciones representacionales sobre la migración paraguaya. Tal como ha sido indicado en la introducción, la pregunta que da origen al análisis de los datos subsiguientes fue formulada de la siguiente manera: "Si tuviera que elegir una palabra que describiera cómo son (en este caso los paraguayos) que viven en la Argentina, ¿qué palabra elegiría?”. El primer dato a analizar es aquel que refiere a la categoría "trabajadores", la cual ha concentrado el 42,4\% de las respuestas (ver Tabla $N^{\circ} 2$ ). Comencemos diciendo que al igual que lo ocurre con la colectividad boliviana, los/as encuestados/as refieren a los paraguayos como parte de la clase trabajadora; en términos clásicos son identificados como obreros o trabajadores manuales. En este sentido, se produce una identificación entre la nacionalidad y el país de procedencia, homologándose uno con otro. Consecuentemente, se identifica con la categoría de trabajadores, ubicando en el imaginario simbólico a los miembros de la colectividad paraguaya en un lugar vinculado a las tareas poco calificadas. Desagreguemos qué matices podemos encontrar dentro de este atributo adjudicado a los paraguayos.

\footnotetext{
${ }^{7}$ Instituto Nacional de Estadística y Censos de la República Argentina

${ }^{8}$ Como se puede ver en la codificación abierta efectuada en la Tabla $N^{\circ} 1$.
} 
En primer lugar, la categoría corresponde exactamente a la palabra que la mayoría eligió para describir a la comunidad en cuestión, incluyendo además un sinónimo de un término del lunfardo nacional "laburadores". No obstante esto, decidimos incluir en dicha categoría también a aquellos que eligieron la palabra "construcción", "albañiles" y "trabajo manual" porque todas refieren a un determinado lugar que se le adjudica a los paraguayos en la estructura productiva del país. También la categoría de "trabajadores" incluye las opiniones que refirieron a los paraguayos como "explotados" y "capaces". Si bien estos fueron atributos menos mencionados, resulta interesante incorporarlos en esta categoría y no en la de "otros" (donde dichas respuestas quedarían invisibilizadas) porque, entendemos, se vinculan con aquella idea de ubicar en el ámbito representacional a los paraguayos en una determinada inserción laboral y de pertenencia a la clase obrera.

\footnotetext{
${ }^{9}$ La palabra "laburadores" forma parte de la jerga argentina. Deviene del término italiano "lavoro" y refiere a personas trabajadoras.
} 
Tabla $N^{\circ}$ 1: Representaciones sociales sobre migrantes paraguayos (categorización “abierta”. Población nativa de 25 a 65 años, CABA, 2014)

REPRESENTACIÓN

TRABAJADORES

ABUSIVOS/TRAICIONEROS

CONSTRUCCION

OTROS

AMABLES/AMISTOSOS

PERSONAS

NS/NC

ETNICIDAD

PARAGUAS

VAGOS

HERMANDAD LATINOAMERICANA

GROSEROS

BORRACHOS

DESCONFIADOS

TOTAL
PORCENTAJE
15,5

10,5

8,5

8

4,7

4,2

4

3,7

2,7

2,3

2

1,7

0,5

100

Fuente: elaboración propia sobre la base del Proyecto de la Universidad de Buenos Aires para Investigación en Ciencia y Técnica (UBACyT), 2012-2014. 
Tabla $N^{\circ}$ 2: Representaciones sociales sobre migrantes paraguayos (categorización “cerrada”. Población nativa de 25 a 65 años, CABA, 2014)

REPRESENTACIÓN

\begin{tabular}{lc}
\hline TRABAJADORES & 42,4 \\
\hline DESVIADOS & 20,2 \\
\hline NS/NC & 11,2 \\
\hline INTEGRABLES & 9 \\
\hline PARAGUAS & 8,7 \\
\hline OTROS & 5,5 \\
\hline ETNICIDAD & 3 \\
\hline TOTAL & 100 \\
\hline
\end{tabular}

PORCENTAJE

.


morales de la sociedad hospitante. Consecuentemente, quebrarían el "contrato" de convivencia establecido entre quienes habitan el territorio.

En suma, los paraguayos son ubicados por los encuestados representacionalmente como migrantes con escasos capitales económicos, al tiempo que sus capitales sociales y culturales son señalados como "diferentes" e "inferiores". La combinación de estas variables, además, es la que explicaría su tendencia a desviarse de las normas. Si la ley es algo creado por "nosotros", en tanto miembros de una comunidad de nacionales, la infracción a esa norma por un sujeto o grupo de sujetos que no forma parte de "nosotros" puede explicarse, desde el punto de vista de los nativos, por su no pertenencia.

La infracción a la norma se ve como una consecuencia natural, que forma parte de la representación negativa elaborada sobre el migrante en cuestión, en los términos de Santamaría (2002) de este "migrante imaginado".

Junto a la categoría "desviados", en la que agrupamos la diversidad de atributos acentuadamente negativos acerca de los paraguayos, creamos la categoría "paraguas", que también refiere a un modo negativo de adjetivar, pero que además implica una mirada condescendiente de los encuestados, es decir, inferiorizante acerca de la personalidad de los paraguayos. En esta categoría incluimos la respuesta literal "paraguas" (que suele ser usada de modo recurrente como apodo para los paraguayos en Argentina) y aquellas como: "sucios", "brutos", "villeros", "no se saben expresar". Todas ellas refieren a un modo de clasificar y calificar a los paraguayos como sujetos con una "cultura" inferior.

No obstante, estas opiniones que mayormente construyen a la comunidad paraguaya de modo negativo, no resulta despreciable que el $9 \%$ haya considerado que los paraguayos son "integrables", dato que se relaciona al ver que solo el $0,2 \%$ piensa que los miembros de la comunidad guaraní son “desconfiados”. Ello podría explicarse, como ya se especificó, por la larga historia y presencia de migrantes paraguayos en el país, que los coloca en una posición de comunidad "conocida" y "cercana" para la sociedad hospitante. Ahora bien, no debe olvidarse que la idea de integración en materia de migraciones también puede suponer la jerarquización de unos y otros. Son los paraguayos los que deben asimilarse a la cultura "hegemónica" de los argentinos. De esta manera, por una parte, la individualidad del extranjero se disuelve en el colectivo, al tiempo que la única posibilidad para el migrante pareciera ser la de la aculturación.

La opción de "NS/NC" (11,2\%) requiere de una clarificación, ya que si bien dicha categoría incluye la respuesta literal: "NS/NC", también contiene la de "personas", que reúne un $4,7 \%$, y la de "hermandad latinoamericana", que concentra un 2,3\%, antes de ser ambas reagrupadas en la categoría "NS/NC" (estos porcentajes sin agrupar se evidencian en la Tabla $\mathrm{N}^{\circ} 1$ ). Entendemos que la palabra "persona" es una respuesta genérica que se asimila a una no respuesta. Algo similar ocurre con la opción "hermandad latinoamericana", en la 
que literalmente aparecen palabras como: "hermano latinoamericano", "vecinos", "blancos", "iguales".

En suma, estas caracterizaciones que construyen los nativos inciden en la constitución de la identidad de los paraguayos, ya que deducimos que la misma es producto de lo que los migrantes recrean en sus vidas cotidianas con sus pautas culturales, sus reivindicaciones políticas, sus vínculos familiares y atributos socioculturales, pero también es el efecto de la mirada de la sociedad hospitante que colabora en la construcción de una identidad paraguaya en Argentina. Tal como plantea Hall (1997), la mirada de los otros también nos instituye $y$, en ocasiones, nos fija, determinando aspectos de nuestra constitución como sujetos.

De esta manera, el análisis de las representaciones sociales sobre los paraguayos evidencia los preconceptos que los nativos tienen acerca de aquellos. Los prejuicios operan por categorización, generalización y desindividualización (Van Dijk, 1997). Las conclusiones a las que se llega por medio de un razonamiento prejuicioso adquieren un estatus de cuasidogma. Por otro lado, los atributos adjudicados a los sujetos trascienden a estos, ramificándose a toda la colectividad. Asimismo, en la acción de representar al migrante

los narradores implementan, realizan y legitiman o cuestionan el conocimiento, las actitudes y las ideologías del grupo y de ese modo contribuyen a la reproducción de los prejuicios (...) que a su vez acentúan la discriminación y, por lo tanto, en forma indirecta la desigualdad. (Van Dijk, 1997, p.164)

Con la reproducción de los prejuicios, se reafirma el lugar de los nativos como dominantes del grupo y se refuerza la posición ocupada por los dominados. Es en el señalamiento de las diferencias con "nosotros" -luego inferiorizadas, negativizadas o simplemente "respetadas" discursivamente- como diferencias naturalizadas, dada la pertenencia nacional, que se asienta la desigualdad. En este sentido, en la mirada de los nativos que hemos podido reconstruir a partir de los resultados del cuestionario, está la idea de que los paraguayos son sujetos fundamentalmente desviados de la norma (violentos y problemáticos), al tiempo que se los vincula con su pertenencia a la clase trabajadora, en potencia "deslealmente" competidora de la fuerza de trabajo nacional. Sobre este último punto volveremos en relación con las representaciones sociales sobre los bolivianos.

\section{Representaciones sociales sobre bolivianos}

En relación con la migración boliviana, se trata en tamaño de la segunda comunidad migratoria de la Argentina, siendo 345.272 personas, según el Censo Nacional de Población, Hogares y Viviendas de 2010, sobre un total de 1.800 .000 extranjeros en la 
Argentina (5\% de la población total). De esta forma, representan el $19 \%$ del total de extranjeros detrás de los paraguayos $(30 \%) .^{10}$

Cabe aclarar que la migración boliviana al igual que la paraguaya constituye una corriente histórica, principalmente hacia zonas fronterizas. Entre 1880 y 1930, los bolivianos llegaron a la Argentina atraídos por la demanda estacional de mano de obra masculina para las cosechas agrícolas en los ingenios azucareros del norte argentino (Sassone, 2007). Sin embargo, entre 1980 y 1990 este colectivo migratorio adquirió una renovada visibilidad al insertarse básicamente en el medio urbano. En ese momento, un gran porcentaje de inmigrantes procedentes de Bolivia arribó a Buenos Aires como consecuencia de la escasez y el deterioro de las tierras, la caída de los precios de los productos regionales, la mecanización del agro, la consolidación del modelo de sustitución de importaciones en la industria y la fuerte crisis laboral dada por la liberalización de la economía, entre otras causas (Caggiano, 2005; Canelo, 2013).

Tal como destacan diversos autores, muchos se han asentado en el Área Metropolitana de Buenos Aires y en la Capital Federal, donde centramos la investigación. Dicho esto, analizaremos las representaciones que sobre ellos han construido los nativos de entre $25 \mathrm{y}$ 65 años residentes en diversos barrios de la Ciudad Autónoma de Buenos Aires encuestados en el estudio mencionado.

Tal como señalamos anteriormente, y al tratarse de una pregunta con respuesta abierta, hemos realizado una primera categorización abierta, siguiendo la teoría fundamentada basada en Glaser y Strauss (1967) y en Strauss y Corbin (2002), y posteriormente hemos cerrado dichas categorías, agrupando las respuestas por afinidad, siguiendo criterios estrictamente teórico-analíticos (ver Tabla $\mathrm{N}^{\circ} 3$ ). La pregunta que da origen al análisis de los datos subsiguientes fue formulada de la siguiente manera: "Si tuviera que elegir una palabra que describiera cómo son (en este caso los bolivianos) que viven en la Argentina, ¿qué palabra elegiría?".

\footnotetext{
${ }^{10}$ Sin embargo, los números aludidos no incluyen el gran porcentaje de migrantes irregulares en cuanto a la situación jurídica para la legislación migratoria argentina.
} 
Tabla $N^{\circ}$ 3: Representaciones sociales sobre migrantes bolivianos (categorización “abierta”. Población nativa de 25 a 65 años, CABA, 2014)

REPRESENTACIÓN

TRABAJADORES

VERDULEROS

SUMISOS

PERSONAS

SUCIO/OLOR

BOLITAS

BONDAD

INVASIÓN

OTROS

$\mathrm{NS} / \mathrm{NC}$

CERRADOS

VAGOS

TOTAL
PORCENTAJE

39,4
9

7,5

7

6,7

6

5

4

2,7

1,7

1,2

100

Fuente: elaboración propia sobre la base de Proyecto de la Universidad de Buenos Aires para Investigación en Ciencia y Técnica (UBACyT), 2012-2014.

Al igual que para el caso de las representaciones respecto de los paraguayos, aquí seguimos, en primer lugar, una codificación abierta, que dio como resultado, a partir del procesamiento de las respuestas, la Tabla $\mathrm{N}^{\circ}$ 4. La categoría que concentró un mayor porcentaje de frecuencias fue la de "trabajadores" con un 39,4\%, seguido por "verduleros" con $9,7 \%$ y "sumisos" con un 9\%. Sin embargo, decidimos posteriormente agrupar estas tres categorías en la de "explotados" (ver Tabla $\mathrm{N}^{\circ} 4$ ), entendiendo que todas ellas aluden a representaciones sociales que asocian a los migrantes de origen boliviano con el trabajo 
arduo, en tareas no calificadas, principalmente en la producción y venta de frutas y verduras, con salarios mínimos y condiciones de trabajo muy duras.

Tabla $N^{\circ}$ 4: Representaciones sociales sobre migrantes bolivianos (categorización “cerrada”. Población nativa de 25 a 65 años, CABA, 2014)

REPRESENTACIÓN

PORCENTAJE

EXPLOTADOS

58,1

BOLITAS

13,7

$\mathrm{NS} / \mathrm{NC}$

10,2

BONDAD

6

INVASIÓN

5

OTROS

4

CERRADOS

1,7

VAGOS

1,2

TOTAL

100

Fuente: elaboración propia sobre la base de Proyecto de la Universidad de Buenos Aires para Investigación en Ciencia y Técnica (UBACyT), 2012-2014.

Tradicionalmente, los migrantes limítrofes tendieron a ocupar "nichos" laborales en ciertos trabajos que los nativos no aceptaban. Al cambiar el horizonte laboral de los argentinos, la nueva situación generó que estos últimos empezaran a competir con los migrantes por los mismos puestos de trabajo (Grimson, 2013). Esta percepción está presente en relación tanto con los bolivianos como con los paraguayos. En este marco, desde los ámbitos estatales se fue constituyendo un discurso que adjudicaba a los inmigrantes limítrofes la responsabilidad por los problemas sociales, económicos, sanitarios y de seguridad del país (Oteiza, Novick y Aruj, 1996). 
Sin embargo, también es necesario considerar el anclaje de las representaciones sociales en la jerarquización y la conformación de "nichos laborales". ${ }^{11}$ En este sentido, Wolf (1993) sostiene que las clasificaciones que diferencian la fuerza de trabajo y que asignan ciertos trabajadores a determinadas posiciones laborales y a otros trabajadores a otras en virtud de sus características culturales o raciales, dan forma a una segmentación étnica del mercado de trabajo que resulta funcional a las actuales modalidades de acumulación del capital. En el caso de los nichos de trabajo destinados a inmigrantes recientes, la adecuación de ciertos inmigrantes para ocupar las posiciones laborales más desfavorables es justificada a través de jerarquías culturales y discriminaciones étnico-nacionales que muchas veces se basan en estereotipos y prejuicios raciales.

Continuando con el análisis, como puede observarse en la Tabla $\mathrm{N}^{\circ} 3$, la categoría que a continuación agrupó mayor cantidad de frecuencias porcentuales fue la de "personas" con un 7,5\% de respuestas. Bajo esa nomenclatura agrupamos aquellas respuestas que referían a los migrantes bolivianos como "personas", "seres humanos", "comunes" y otras palabras que, consideramos, forman parte de un discurso que, tras el ocultamiento de la mención de alguna característica, pretende ser políticamente correcto. ${ }^{12}$ Por este motivo, al no dar ningún tipo de respuesta ante la pregunta que solicita vincular la migración con alguna palabra, decidimos unificarlo con la categoría "No sabe/No contesta", entendiendo que ambas forman parte de una misma lógica de evasión. De ahí que en la Tabla $\mathrm{N}^{\circ}$ 4, la categoría "NS/NC" alcanza el 10,2\% de respuestas, un porcentaje más alto que en la Tabla $\mathrm{N}^{\circ} 3$.

Otra representación con un alto porcentaje de respuestas fue la de "Sucio/Olor" con un 7\%, seguido por "bolitas" con un 6,7\%. Si bien ambas denominaciones podrían ser pensadas como elementos muy distintos, aludiendo una a falta de higiene y la otra a una forma despectiva de referirse a los inmigrantes de origen boliviano, decidimos unificarlas bajo la categoría "bolitas", constituyendo conjuntamente el 13,7\% de las respuestas, tal como puede observarse en la Tabla $\mathrm{N}^{\circ}$ 4. La razón de esto reside en que consideramos que tal forma de referirse a este colectivo migratorio incluye las connotaciones negativas como la falta de higiene, la falta de cuidado y la referencia al otro como un ser inferior. Al igual que la categoría "paraguas" para las representaciones hacia migrantes paraguayos, la categoría "bolita" también entraña un modo de adjetivar negativamente y con connotaciones inferiorizantes.

La categoría "bondad", que en ambas tablas concentra un 6\% de las respuestas, no la agrupamos con otra categoría por ser la única que refiere a un aspecto positivo con el que

\footnotetext{
${ }^{11}$ Herrera Lima (2005) se refiere con este concepto a "nichos de mercado en actividades económicas que de hecho no podrían existir y renovarse a lo largo del tiempo si no fuera por la presencia renovada de sucesivas olas de inmigrantes" (p. 171).

${ }^{12}$ Esta temática ha sido ampliamente estudiada en la aplicación de cuestionarios bajo el concepto de "sesgo de deseabilidad social". Ver Cea D'Ancona $(2005,2009)$.
} 
se representa a esta corriente migratoria, aunque podría pensarse junto con la sumisión, es decir, una bondad que tiene connotaciones "torpes", "inocentes".

"Invasión" es otra categoría con un porcentaje de 5\% en ambas tablas, la que no hemos agrupado junto a otra ya que hace referencia a la presencia numéricamente importante de bolivianos, representación que, consideramos, ha sido construida con gran ayuda de los medios masivos de comunicación y discursos políticos que datan de mediados de la década de 1990, cuando personas de origen boliviano se trasladaron hasta las ciudades, especialmente a determinados barrios del Área Metropolitana del Gran Buenos Aires y la Ciudad Autónoma de Buenos Aires.

Esta imagen de la invasión es explicada por Grimson (2013) aludiendo al cambio en el régimen de visibilidad de la etnicidad en la Argentina, que pasó de una situación de invisibilización de la diversidad a una creciente hipervisibilización de las diferencias, lo que se combinó a la vez con los cambios sociodemográficos anteriormente mencionados, como el desplazamiento de migrantes bolivianos hacia centros urbanos.

Con porcentajes muy menores encontramos las categorías "cerrados" y "vagos" (1,7\% y $1,2 \%$, respectivamente), consideradas ambas como representaciones también negativas hacia los migrantes bolivianos, tal como hemos observado con la mayoría de las respuestas analizadas anteriormente.

De esta forma, podemos concluir que las miradas hacia la migración boliviana concentran características mayoritariamente negativas, asociándolas principalmente al trabajo, pero siendo considerada una competencia desleal frente a la mano de obra nativa, por someterse a peores condiciones laborales, contribuyendo de esta forma al desempleo del cual se los culpabilizó en los discursos mediáticos de fines de los años noventa y principios de 2000.

Sin embargo, no son asociados a la violencia ni la delincuencia, como sí sucede con la migración paraguaya, sino que predominan las representaciones sociales que los etnitizan y esencializan, colocándolos en el lugar de la pasividad y la aceptación de la explotación.

\section{Representaciones sociales sobre argentinos}

Finalmente nos proponemos analizar las representaciones que los argentinos construyen en relación con su propia identidad, o bien, lo que algunos autores denominan "notridad", es decir, las representaciones sociales que los argentinos (particularmente aquellos que residen en la Ciudad Autónoma de Buenos Aires) elaboran acerca de los atributos (individuales y colectivos) y elementos culturales que definirían su identidad como grupo. Estos atributos "potencian un sentimiento de conexión con otros miembros de la comunidad cuya construcción simbólica depende de, y al mismo tiempo favorece, el desarrollo de una identidad unificadora" (Alexander cit. en Terrén, 2003, p. 134). 
Cabe aclarar que entendemos la identidad como una construcción continua y nunca definitiva en la que se intersectan valores, pautas culturales, vivencias compartidas, expectativas a futuro y reinterpretaciones del pasado reciente e histórico. El conjunto de estos elementos identitarios son reinterpretados en el presente por los miembros del colectivo nacional, constituyendo así un imaginario acerca del "ser nacional" que se naturaliza y se presenta como "evidente". A continuación, presentamos las categorías construidas inicialmente sobre la base de las respuestas obtenidas, la distribución de frecuencias porcentuales correspondientes a la pregunta: "Si tuviera que decir cuál es la característica de ser argentino, lo que nos identifica como país, ¿qué diría?” (Tabla $\mathrm{N}^{\circ}$ 5), así como la elaboración final de las categorías (Tabla $\mathrm{N}^{\circ}$ 6).

Tabla $N^{\circ}$ 5: Representaciones sociales sobre "ser argentinos" (categorización abierta. Población nativa, 25 a 65 años. CABA, 2014)

REPRESENTACIÓN

REPRESENTACIÓN ICONOGRÁFICA

SOBERBIA/ARROGANCIA

VENTAJISTA/"CHANTA"

SOLIDARIDAD

ADAPTABILIDAD/TENACIDAD

FAMILIEROS/AMIGUEROS

SENSIBILIDAD

RECEPTIVOS A LA MIGRACIÓN

OTROS

NS/NC

INDIVIDUALISMO

TOTAL

\section{PORCENTAJE}

17,5

13,2

12,2

8,5

8

6,7

5,7

5

2,7

2,5

100

Fuente: elaboración propia sobre la base de Proyecto de la Universidad de Buenos Aires para Investigación en Ciencia y Técnica (UBACyT), 2014. 
Tabla $N^{\circ}$ 6: Representaciones sociales sobre "ser argentinos" (categorización cerrada. Población nativa, 25 a 65 años. CABA, 2014)

REPRESENTACIÓN

PORCENTAJE

IDIOSINCRACIA

ABIERTOS

SOBERBIA/ARROGANCIA

INDIVIDUALISTAS

ADAPTABILIDAD/TENACIDAD

SENSIBILIDAD

OTROS

$\mathrm{NS} / \mathrm{NC}$

TOTAL
26

17.9
15.7

8.5

6.7

5

2.7

100

Fuente: elaboración propia sobre la base de Proyecto de la Universidad de Buenos Aires para Investigación en Ciencia y Técnica (UBACyT), 2014.

Al analizar la Tabla $\mathrm{N}^{\circ} 6$ podemos observar que la mayor cantidad de frecuencias porcentuales se concentran en la categoría que hemos denominado "idiosincracia" (26\%). Bajo esta nomenclatura hemos agrupado aquellas respuestas que aludían a aspectos vinculados a imágenes, objetos y actividades que son considerados popularmente como representativos de la "argentinidad", entre cuyas respuestas podemos mencionar el mate, el fútbol y la carne. Estas respuestas consideramos que son neutras, en el sentido de que no expresan una valoración positiva o negativa, sino que simplemente dan cuenta de un imaginario común, una serie de gustos y actividades que compartirían los argentinos, vistos en un conjunto imaginado. También en esta categoría hemos incluido aquellas respuestas que aluden a los valores familiares y de amistad que los/as encuestados/as autoperciben como marca significativa de la identidad nacional.

Se trata de lo que Terán (2008) denomina "el nacionalismo culturalista", cuando explica que "el nacionalismo culturalista dice que ser argentino o lo que fuere es identificarse con un conjunto de pautas culturales (tales como la lengua, ciertos símbolos, usos y costumbres presentes y pasados, cierto «tipo nacional»)" (p. 121). Con ello alude a elementos y 
símbolos que reafirman la pertenencia a un grupo mayor, en este caso, el ser argentino. En la misma línea, Anderson (1993) postula que la nación constituye

una comunidad política imaginada porque aún los miembros de la nación más pequeña no conocerán jamás a la mayoría de sus compatriotas, no los verán ni oirán siquiera hablar de ellos, pero en la mente de cada uno vive la imagen de su comunión. (p. 23)

En las representaciones sociales de los/as encuestados/as parece entreverse un lazo común invisible que recorre a la población de origen argentino. Sería este origen común el que hace compartir gustos, especialmente culinarios y recreativos, que los aúna en un grupo con características comunes, y que los diferenciaría de aquellos que no forman parte del mismo. Como ya lo observara Hall (1996), la identificación se basa en un proceso de reconocimiento de cierto origen común, de atributos o ideales compartidos y en el establecimiento de un vallado natural, de un lazo a partir del que se edifica un vínculo de lealtad entre los miembros de la comunidad.

La categoría que hemos denominado "abiertos" reúne un 17,9\% de respuestas. En ella incluimos las representaciones ligadas a la solidaridad y la apertura a la inmigración. Vinculamos ambos aspectos en la misma categoría, ya que la pregunta se encontraba a continuación de un bloque de preguntas sobre la temática migratoria y, en muchos casos, las respuestas hacían alusión a la narrativa dominante de la nación argentina como un país abierto, donde históricamente han sido recibidos con beneplácito los extranjeros provenientes de ultramar. De acuerdo con los estudios realizados por Sergio Caggiano (2005), los proyectos de la generación del 37 y, más tarde, los de la del 80, postulaban que la Argentina debía ser una nación abierta a la inmigración, tal como ha sido plasmado en el preámbulo de la Constitución Nacional de 1853. Este mito estuvo asociado a otro, el de la integración. Es decir, se trataba de una política no solo proinmigratoria, sino también de integración de esa inmigración, con un gran sesgo asimilacionista.

Con un porcentaje muy similar (17,5\%) se concentran las respuestas que hicieron alusión al carácter "soberbio o arrogante" que tendrían los argentinos. Al respecto, si bien solo lo planteamos a modo de hipótesis para seguir profundizando en otros trabajos, se trata de una característica vinculada a los orígenes españoles e italianos que supuestamente tendrían la gran mayoría de los argentinos, en concordancia con el mito de "ser una nación que desciende de los barcos".

Planteamos entonces que la representación del ser soberbio/arrogante se vincula al imaginario construido acerca de la "creencia de la excepcionalidad argentina", compuesta por una población mayoritariamente europea, presente en los discursos de la época de construcción del Estado Nacional, tales como los de Alberdi y Sarmiento. En este período, las migraciones cumplieron una función primordial. Como mencionara Pellegrino (2000), el propósito principal y explícito de los gobernantes argentinos no era solamente el de "poblar el desierto", sino también el de modificar sustancialmente la composición de su 
población, sumando a la población nativa la de inmigrantes europeos, que debían transmitir sus valores al conjunto de los habitantes del país. Ellos introducirían hábitos de orden y de buena educación, hábitos de industria y de laboriosidad, y los transmitirían al conjunto de la población. Estas creencias, consideramos, que dan origen a las autorrepresentaciones de los argentinos como una "población superior" a la del resto de los países latinoamericanos, dada su mayoritaria "imaginada" blanquedad (Crosa Portilli, Silberstein y Tavernelli, 2009).

Con un porcentaje del 15,7\%, se encuentran agrupadas las respuestas en la categoría "individualistas". Bajo este nombre concentramos aquellas palabras que hacen alusión a una supuesta característica que definiría al "ser" argentino, signado por una actitud que suele adscribirse a la población que vive en Buenos Aires, y que consiste en lo que algunas personas denominan "viveza criolla", es decir, conseguir aquello que se desea de una forma facilista, que no requiere mucho esfuerzo en función de un objetivo o fin de provecho individual. Pero también la palabra chanta, según la Real Academia Española (RAE), hace referencia a aquella persona "que presume de tener algo, especialmente una capacidad, un conocimiento o un poder, que en realidad no posee". Esta es una característica que suele atribuirse a -pero de la que también hacen gala- los porteños ${ }^{13}$.

La categoría "adaptabilidad/tenacidad" contiene el 8,5\% de las respuestas. La misma ha sido referida, en la mayoría de los casos, a la capacidad que tendrían los argentinos para salir adelante en momentos de crisis, estando sobre todo muy presente en el imaginario la crisis económico-político-social de $2001^{14}$.

De esta forma, las construcciones representacionales respecto de la identidad argentina o de la "notridad" contienen elementos positivos y negativos, siendo preponderante el imaginario de una población homogénea, en su mayoría, descendiente de los barcos europeos.

\footnotetext{
${ }^{13} \mathrm{Si}$ bien este término se utiliza para aludir a aquella persona que vive en una ciudad que tiene puerto, en el caso de la Argentina hace referencia a aquellos que viven en la ciudad portuaria de Buenos Aires.

${ }^{14}$ La crisis de diciembre de 2001 en Argentina, o crisis de 2001, también referida como el "Cacerolazo" o el "Argentinazo", fue una crisis política, económica, social e institucional, potenciada por una revuelta popular generalizada bajo el lema "¡Qué se vayan todos!". Esta crisis causó la renuncia del entonces presidente de Argentina, Fernando de la Rúa, dando lugar a un período de inestabilidad política durante el cual cinco funcionarios ejercieron la Presidencia de la Nación. Sucedió en el marco de una crisis mayor que se extendió entre 1998 y 2002, originada por una larga recesión que conllevó una crisis humanitaria, de representatividad, social, económica, financiera y política (Telechea, 2006). El desencadenante inmediato de la crisis fue la imposición del "Corralito", una disposición del gobierno que restringía la extracción de dinero en efectivo de los bancos, diseñada por el entonces ministro de Economía, Domingo Cavallo. La revuelta se generalizó el 19 de diciembre de 2001, inmediatamente después de que el presidente radical Fernando de la Rúa anunciara el establecimiento del estado de sitio, hecho que originó su renuncia al día siguiente. La mayor parte de las personas que participaron en las protestas fueron autoconvocadas y no respondían a ningún partido político, sindicato u organización social estructurada.
} 


\section{Conclusiones}

A lo largo del presente escrito nos propusimos analizar las representaciones que la población nativa construye en relación con la población de origen boliviano y paraguayo, así como en relación con la propia identidad nacional. Para ello, retomamos las preguntas abiertas relevadas en un cuestionario aplicado a 401 personas nativas residentes en la Ciudad Autónoma de Buenos Aires, cuyas edades oscilaban entre los 25 y 65 años. Sus respuestas fueron procesadas y analizadas a partir de la construcción de un índice conceptual, así como mediante una categorización abierta y axial, siguiendo los aportes de Glaser y Strauss,(1967) y Strauss y Corbin (2002).

Consideramos que el trabajo con las representaciones sociales es complejo, y por ello exige la combinación de diversas técnicas y perspectivas, sin ser estas necesariamente antagónicas. Es decir, no partimos de una visión dicotómica entre perspectivas cuantitativas y cualitativas, sino que la elección de uno u otro enfoque dependerá de los objetivos de la investigación.

En esta oportunidad, la aplicación de un instrumento estructurado nos ha permitido acceder a una población mayor, y aplicar un cuestionario extendido que abarcaba diversidad de temas que no podría haber sido posible mediante entrevistas en profundidad. Asimismo, la aplicación del instrumento a una muestra no intencional nos ha permitido obtener cierta representatividad de las respuestas. El conocimiento previo del equipo sobre las relaciones interculturales entre migrantes y nativos en diversos ámbitos es lo que nos ha posibilitado construir un instrumento estructurado de este tipo, y modificar el abordaje metodológico que a lo largo de diez años venía siendo de corte cualitativo a partir de la aplicación de focus groups y entrevistas en profundidad.

De esta forma, utilizamos un instrumento de registro cuantitativo, aunque seleccionamos para este trabajo solo algunas preguntas con formato "abierto" que permiten mayor espontaneidad en la respuesta del encuestado, captando el sentido del discurso, sin estar este precodificado por el investigador.

Para cerrar las respuestas abiertas, decidimos tomar como modelo algunos aportes de la teoría fundamentada, entendiendo que los enfoques metodológicos pueden ser leídos como una caja de herramientas -siempre con un cuidado epistemológico- de modo tal de alcanzar los objetivos propuestos. Siguiendo a los autores de esta escuela, procedimos primero a abrir las categorías, para, posteriormente, cerrarlas mediante una construcción conceptual.

Hemos considerado que la categoría de representaciones sociales resultaba pertinente como herramienta de análisis, ya que su potencialidad se basa en vincular los imaginarios sociales con las prácticas, aunque no suceda esto de una manera mecánica. Es decir, si bien acciones e ideas son claramente planos diferentes del análisis, la categoría de representación social posibilita establecer vínculos entre uno y otro elemento, ambos centrales para la 
comprensión de las relaciones que se establecen entre los miembros de las sociedades hospitantes y los migrantes.

En relación con las representaciones construidas sobre bolivianos y paraguayos, consideramos que prevalece un reduccionismo economicista, que circunscribe la problemática de los inmigrantes a su potencialidad como trabajadores y que de esta forma opera definiendo sus posiciones laborales en la sociedad de destino, en el marco de la lógica jerarquizadora del racismo (Wolf, 1993). Asimismo, las migraciones y los migrantes son pensados casi de modo exclusivo a partir de una lógica funcional y/o instrumental.

Del análisis de las representaciones sociales de los argentinos encuestados podemos concluir también que a todos los migrantes (ello incluye por supuesto a los bolivianos y paraguayos sobre los que hicimos referencia) los une su calidad de "extraños". La extrañeza está ligada a un suceso inesperado, repentino y desestructurante. La extrañeza supone el establecimiento de una relación social, ya que no hay extraños en sí mismos, sino extraños para alguien (Sabido Ramos, 2009). En este caso, ese alguien está compuesto por los miembros de la sociedad hospitante, por lo que contemplamos que era importante también analizar las representaciones que los/as encuestados/as - en calidad de nativos/as- elaboran acerca de sí mismos/as.

Las representaciones sociales sobre la "identidad nacional" se apoyan en una serie de elementos comunes, en donde se intersectan fragmentos del pasado, del presente y del futuro, ya que consideramos las identidades como procesos cambiantes y no estáticos. De esta forma, la doble ilusión de la identidad nacional remite a la idea de "proyecto" y de "destino" que compartirían todos los miembros de la comunidad nacional (Balibar, 2005). En este sentido, una nación es una comunidad simbólica (Hall, 1997).

En otros términos, la información acerca de quiénes somos, construida grupalmente, se configura en un campo de representación, de manera ordenada y jerarquizada, que califica y clasifica a la "notridad" y a la "otredad". De esta forma, se tornan pensables y analizables ciertas conductas y la producción de determinadas relaciones sociales.

Nos propusimos entonces aprehender el proceso por el cual los nativos piensan a otros grupos y a sí mismos. Lo simbólico constituye nuestra realidad social, es decir, las representaciones sociales son producto de lo que acontece en el mundo social, al tiempo que este último es un reflejo de aquellas.

En suma, la idea que atraviesa el presente artículo remite a que el "vocabulario no es jamás una herramienta neutra" (Baczko, 1999, p.17). Las palabras califican, clasifican y estructuran el modo en que somos vistos y vemos a los demás. Al nombrar excluimos y también incluimos, repercutiendo en el proceso de constitución de las identidades.

\section{Referencias bibliográficas}


Aliaga Sáez, F. A., Maric Palenque, M. L y Uribe Mendoza, C. J. (2018). Imaginarios y representaciones sociales: Estado de la investigación en Iberoamérica. Bogotá: Universidad Santo Tomás.

Anderson, B. (1993). Comunidades imaginadas. Reflexiones sobre el origen y la difusión del nacionalismo. México: Fondo de Cultura Económica.

Baczko, B. (1999). Los imaginarios sociales: memorias y esperanzas colectivas. Buenos Aires: Ed. Nueva Visión.

Balibar, E. (2005). Violencias, identidades y civilidad. Barcelona: Gedisa.

Banchs, M. (2000). Aproximaciones procesuales y estructurales al estudio de las representaciones sociales. Papers on Social Representations, 9, 3.1-3.15. Recuperado de http://www.psr.jku.at/PSR2000/9_3Banch.pdf

Berger, T. y Luckmann, P. (2006). La construcción social de la realidad. Buenos Aires: Amorrortu.

Caggiano, S. (2005). Lo que no entra en el crisol. Inmigración boliviana, comunicación intercultural y procesos identitarios. Buenos Aires: Prometeo Libros.

Canelo, B. (2013). Fronteras internas. Migración y disputas espaciales en la Ciudad de Buenos Aires. Buenos Aires: Antropofagia.

Carballude, A. M., Malegaríe, J., Pérez, F. y Santamaría, R. (2004). Puertas adentro: la inmigración discriminada, ayer y hoy. En N. Cohen (comp.), Documentos de trabajo $N^{\circ} 36$. Buenos Aires: Instituto de Investigaciones Gino Germani, Facultad de Ciencias Sociales, Universidad de Buenos Aires. Recuperado de https://core.ac.uk/download/pdf/35145927.pdf

Cea D' Ancona, M. A. (2005). La exteriorización de la xenofobia. REIS, 112, 197-203.

Cea D' Ancona, M. A. (2009). La compleja detección del racismo y la xenofobia a través de encuestas. Un paso adelante en su medición. Revista Española de Investigaciones Sociológicas, $125, \quad 13-45 . \quad$ Recuperado de http://www.reis.cis.es/REIS/PDF/REIS_125_011231144723167.pdf

Crosa Pottilli, J., Silberstein, Y. y Tavernelli, R. (2009). De la jerarquización de naciones a la clasificación de sujetos: representaciones que perpetúan un orden exclusor. En N. Cohen (ed.), Representaciones de la diversidad: trabajo, escuela y juventud (pp. 3064). Buenos Aires: Ediciones Cooperativas.

Devoto, F. (2003). Historia de la inmigración en la Argentina. Buenos Aires: Sudamericana.

Durkheim, E. (2000). El suicidio. Buenos Aires: Editorial Bitácora. 
Elias, N. (2003). Ensayo acerca de las relaciones entre establecidos y forasteros. REIS, 104, 219-251. DOI: $10.2307 / 40184576$

Glaser, G., y Strauss, A. (1967). The discovery of grounded theory. Strategies for qualitative research. Londres: Aldine Publishing Company.

Grimson, A. (2013). Relatos de la diferencia y la igualdad. Los bolivianos en Buenos Aires. Buenos Aires: Eudeba.

González, A. y Kleidermacher, G. (2018). Representaciones sociales de la "identidad argentina" en la población nativa residente en la Ciudad Autónoma de Buenos Aires. Civilizar, 18(34), 135-148. Recuperado de https://doi.org/10.22518/usergioa/jour/ccsh/2018.1/a09

Hall, S. (1996). ¿Quién necesita identidad? En S. Hall, y P. Du Gay (eds.), Cuestiones de identidad cultural. (pp. 1-39). Buenos Aires: Amorrortu.

Hall, S. (1997). Sin garantías. Trayectorias y problemáticas en estudios culturales. Popayán-Lima-Quito: Envión Editores-IEP- Instituto Pensar-Universidad Andina Simón Bolívar.

Halpern, G. (2009). Etnicidad, inmigración y política. Buenos Aires: Prometeo.

Herrera Lima, F. (2005). Vidas itinerantes en un espacio laboral transnacional. México: Universidad Autónoma Metropolitana.

Instituto Nacional de Estadísticas y Censos. (2010). Censo Nacional de Población, Hogares y viviendas. Buenos Aires: Instituto Nacional de Estadísticas y Censos.

Jodelet, D. (1991). Representaciones sociales, un área en expansión. En D. Páez, C. Sanjuan, I. Romo y A. Vergara (eds.). Sida: imagen y prevención (pp. 25-30). Madrid: Fundamentos.

Mckinney, J. (1968). Tipología constructiva y teoría social. Buenos Aires: Editorial Amorrortu.

Moscovici, S. (1986). Psicología social II: Pensamiento y vida social, psicología social y problemas sociales. Barcelona: Paidós.

Oteiza, E., Novick, S. y Aruj, R. (1996). Inmigración y discriminación: políticas y discursos. Buenos Aires: Grupo Editor Universitario.

Pellegrino, A. (2000). Migrantes latinoamericanos, síntesis histórica y tendencias recientes. Montevideo: Universidad de la República y Centro Latinoamericano y Caribeño de Demografía (CELADE-CEPAL).

Sabido Ramos, O. (2009). El extraño. En E. León Vega (ed.), Los rostros del otro (pp. 2557). Barcelona: Anthropos. 
Santamaría, E. (2002). La incógnita del extraño. Barcelona: Anthropos.

Sassone, S. (2007). Migración, territorio e identidad cultural: construcción de lugares bolivianos. Población de Buenos Aires, 4(6), 9-28.

Strauss, A. y Corbin, J. (2002). Bases de la investigación cualitativa. Técnicas y procedimientos para desarrollar la teoría fundamentada. Bogotá, Colombia: CONTUS-Editorial Universidad de Antioquía.

Telechea, R. (2006). Historia de los cacerolazos: 1982-2001. Razón y Revolución, 16, segundo semestre, 141-184. Recuperado de http://revistaryr.org.ar/index.php/RyR/article/view/171/162

Terán, O. (2008). Historia de las ideas en la Argentina. Diez lecciones iniciales, 18101980. Buenos Aires: Siglo XIX.

Terrén, E. (2003). La ironía de la solidaridad: cultura, sociedad civil y discursos sobre el conflicto racial de El Ejido. REIS, 102, 125-146. DOI: 10.2307/40184539

Van Dijk, T. (1997). Historias y racismo. En D. Mumby (ed.), Narrativa y control social. Perspectivas críticas (pp. 163-189). Buenos Aires: Amorrortu.

Vernik, E. (2004). Qué es una Nación. La pregunta de Renán revisitada. Buenos Aires: Prometeo.

Wolf, E. (1993). Europa y la gente sin historia. México: Fondo de Cultura Económica 